\title{
Welche Ärzte am häufigsten verklagt werden
}

\section{Ärztliche Tätigkeit in den USA ist risikoträchtig. Je nach Facharztdisziplin müssen 75 bis $99 \%$ aller Ärzte bis zum 65. Lebensjahr mit mindestens einer Schadenersatzforderung rechnen.}

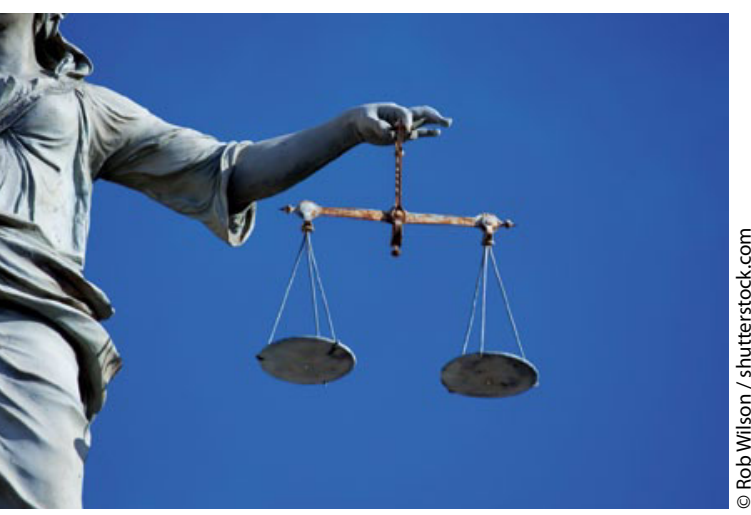

D ie Autoren durchsuchten die Archive einer großen Versicherung nach allen Schadenersatzforderungen in den Jahren 1991-2005. Jedes Jahr müssen 7,4\% aller Ärzte mit einer Schadenersatzklage rechnen, doch kommt es nur bei 1,6\% zu Zahlungen, d. h. 78\% der Kläger bleiben erfolglos. Unter 25 Facharztdisziplinen gibt es ein bemerkenswertes Gefälle: Während jedes Jahr etwa 19,1\% der Neuro-, 18,9\% der Herz-Lungen- und 15,4\% der Allgemeinchirurgen, also nahezu jeder Fünfte mit einer Klage rechnen muss - stehen am anderen Ende die Psychiater (2,6\%), Pädiater $(3,1 \%)$, Allgemeinmediziner $(5,2)$ und Dermatologen (5,5\%).

Die Zahlungen lagen im Mittel bei 274.887 US-\$, bei Pädiatern mit 520.923 US-\$ am höchsten und bei Dermatologen mit 111.832 US-\$ am niedrigsten. Inter- nisten und Allgemeinmediziner liegen bei der Zahl der Klagen an 14. bzw. 22., bei der Summe der Zahlungen (median) an dritter bzw. elfter Stelle.

Jena $A B$ et al. Malpractice risk according to phycisian speciality. New Engl J Med 2011; 365: 629-36

Kommentar: Alle Anzeichen deuten darauf hin, dass wir in Deutschland zwar noch keine amerikanischen Verhältnisse haben, was die Zahl der Klagen und die Höhe der Entschädigungen angeht. Doch nehmen auch bei uns die Schadenersatzklagen zu. Einige Ergebnisse aus den USA dürften Allgemeingültigkeit besitzen. Das Risiko für Klagen ist zwar ausgesprochen facharztspezifisch, doch die Zahl der Klagen korreliert nicht mit der Höhe der Entschädigungen. Beispielsweise haben Geburtshelfer und Allgemeinchirurgen ein hohes, Pädiater und Pathologen ein geringes Risiko für Klagen, doch bei den Entschädigungen sind die Summen für Pädiater und Pathologen höher als bei Geburtshelfern und Allgemeinchirurgen. Es ist bemerkenswert, dass drei von vier Klagen nicht zum Erfolg führen. Doch zur Beruhigung trägt diese Tatsache nicht bei: Jeder Arzt kann sich gegen die direkten Kosten versichern. Doch wird er bei ungerechtfertigten Klagen die indirekten Kosten wie Zeitaufwand, Stress, zusätzliche Arbeit und Imageschaden selbst meistern müssen. Prof. Dr. Heinrich Holzgreve

\section{Schädel-Hirn-Trauma: Was deutet auf Misshandlung hin?}

Bei einer Misshandlung sind Apnoe, retinale Blutungen, Rippenfrakturen, Langknochenfrakturen, Krampfanfälle und Kontusionen an Kopf oder Hals häufiger als bei anderen Schädelhirntraumata (SHT). In einer Studie wurden Fälle von 1.053 Kindern mit SHT, davon 348 nach Misshandlung (,abusive head trauma“, AHT) ausgewertet. Retinablutungen (häufig) und Rippenfrakturen (selten) waren mit dem höchsten AHT-Risiko verbunden. Während ein Kind mit SHT ohne einen der oben genannte Befunde ein AHTRisiko von $4 \%$ hatte, stieg dieses Risiko auf $58 \%$, wenn eine Retinablutung bestand. Wurde außer einer retinalen Hämorrhagie oder einer Rippenfraktur noch einer der vier anderen Befunde erhoben, stieg die Wahrscheinlichkeit für Missbrauch auf über $85 \%$. Jede Kombination von mindestens drei der klinischen Zeichen hatte einen positiven Vorhersagewert von über $85 \%$. Dr. Beate Schumacher

Maguire SA et al. Pediatrics 2011; 128: e550 